\title{
MicroRNA-155 promotes the proliferation and invasion abilities of colon cancer cells by targeting quaking
}

\author{
BIN HE, SHENG-QIANG GAO, LI-DONG HUANG, YUE-HAN HUANG, QI-YU ZHANG, \\ MENG-TAO ZHOU, HONG-QI SHI, QI-TONG SONG and YUN-FENG SHAN \\ Department of Hepatobiliary Surgery, The First Affiliated Hospital of Wenzhou Medical University, \\ Wenzhou, Zhejiang 325000, P.R. China
}

Received April 5, 2014; Accepted November 5, 2014

DOI: $10.3892 / \mathrm{mmr} .2014 .2994$

\begin{abstract}
The increasing expression of microRNA-155 (miR-155) and decreasing expression of RNA-binding protein quaking $(\mathrm{QKI})$ in colon cells have been observed previously. In this study, we attempted to establish the correlation between miR-155 and QKI. In addition, we assessed whether the expression of miR-155 and QKI is linked to the proliferation and invasion capabilities of colon cells. Firstly, nineteen tumor samples, divided into two groups according to the presence or absence of lymphatic metastasis, were obtained from colon cancer patients at the First Affiliated Hospital of Wenzhou Medical University, China. The expression level of miR-155 and QKI was measured by quantitative polymerase chain reaction (qPCR). Secondly, the GES-1, SW480 and COLO205 cell lines were cultured and the expression level of QKI and miR-155 was also assessed by qPCR. Thirdly, a luciferase reporter gene assay was performed to detect the association between miR-155 and QKI, and qPCR and western blot analysis were performed to confirm the effects of miR-155 on the expression of QKI at the mRNA and protein level. Subsequently, the SW480 cells were used in the following experiments. Following treatment with miR-155 inhibitor and QKI overexpression vector, western blot analysis, propidium iodide (PI) staining and a cell scratch assay were carried out to assess the effects of miR-155 on the proliferation and invasion potential of colon cancer cells. qPCR findings revealed higher miR-155 expression and lower QKI expression in colon cancer tissues as well as the colon cancer cell lines SW480 and COLO205. The relative luciferase activity of the 3 ' untranslated region (3'UTR) was decreased by approximately $45 \%$ when SW480 cells stimulated by mimic-miR-155 were combined
\end{abstract}

Correspondence to: Professor Yun-Feng Shan, Department of Hepatobiliary Surgery, The First Affiliated Hospital of Wenzhou Medical University, 2 FuXue Lane, Wenzhou, Zhejiang 325000, P.R. China

E-mail: yunfengdoc@163.com

Key words: microRNA-155, colon cancer, quaking, proliferation, invasion, SW480 cells with the wild-type 3'UTR constructs. In addition, when the cells were treated with mimic-miR-155, QKI expression was significantly decreased at the mRNA and protein level. These outcomes revealed that miR-155 decreased the production of QKI by acting on the 3'UTR of the QKI gene. Furthermore, PI staining and the cell scratch assay revealed that miR-155 influenced the cell cycle and invasion abilities of colon cancer cells by directly targeting QKI and decreased the production of QKI by acting on the 3'UTR of the QKI gene. This study has demonstrated the correlation between miR-155 and QKI, in which miR-155 regulates the cell cycle and invasion ability of colon cancer cells via the modulation of QKI expression. Our study provides novel therapeutic strategies for colon cancer therapy.

\section{Introduction}

Colon cancer is one of the most common types of cancer in the world, yet its underlying development mechanism is unknown. However, there are a number of indications regarding the progression from colonic epithelial cells to carcinoma. Unhealthy eating habits and environmental pollution aggravate the mutation of genes controlling cell adhesion and proliferation and consequently play significant roles in cancer development (1).

microRNAs (miRNAs) are a class of approximately 22-nt, non-coding RNAs, that generally suppress gene expression through binding to the $3^{\prime}$ untranslated region (3'UTR) of target genes (2-4). microRNA-155 (miR-155) participates in various aspects of cell physiology: it plays a critical role in the innate and acquired immune response and is associated with various diseases including autoimmune disorders, leukemia, atherosclerosis and tumors including breast and lung (5).

In previous studies, high expression of miR-155 in colorectal cancer has been linked to lymph node metastases $(6,7)$. Furthermore, lymph node metastases have long been associated with higher proliferation and invasion abilities. At the same time, a type of RNA-binding protein named quaking (QKI), which suppresses gastric cancer cell proliferation by decreasing $\beta$-catenin expression (8), has been detected in colon cancer (9). The phenomenon of high miR-155 expression and low QKI expression in colon cancer prompted us to investigate the correlation between them and further ascertain whether miR-155 affects the differentiation of colon cancer by targeting QKI directly. 


\section{Materials and methods}

Tissues. The study was approved by the ethics committee of Wenzhou Medical University (Wenzhou, China). After obtaining their informed consent, colon tumors and adjacent normal tissues were collected from surgical patients at the First Affiliated Hospital of Wenzhou Medical University prior to the operation. When cancerous tissues were removed, they were frozen immediately at $-80^{\circ} \mathrm{C}$. Next, the samples were divided into two groups depending on the presence of lymphatic metastasis. The non-invasion group refers to primary colon cancer samples without lymphatic metastasis, while the invasion group refers to cancer samples with lymphatic metastasis.

Cell culture. Human colon cancer cell lines SW480 and COLO205, and the non-malignant gastric intestinal epithelial cell line GES-1 were all cultured in high-glucose Dulbecco's modified Eagle's medium (DMEM; Gibco, Carlsbad, CA, USA) supplemented with $10 \%$ fetal bovine serum (Gibco). Cells were maintained at $37^{\circ} \mathrm{C}$ under a $5 \% \mathrm{CO}_{2}$ atmosphere. SW480 was selected as the cell type for the remaining experiments.

Quantitative polymerase chain reaction $(q P C R)$. $\mathrm{qPCR}$ for mRNA or miRNA analyses was performed using ABI PRISM ${ }^{\circledR}$ 7900HT (Applied Biosystems, Carlsbad, CA, USA), with the following reactions: $95^{\circ}$ for $30 \mathrm{sec}, 40$ cycles of $95^{\circ}$ for $5 \mathrm{sec}$ and $65^{\circ}$ for $34 \mathrm{sec}$. The mRNA levels of 18S and U6 were used as an internal control for mRNA or miRNA, respectively. Relative expression of RNA was measured using the $2^{-\Delta \Delta C}$ method. The primers were as follows: QKI: F-AAGCCC ACCCCAGATTACCT, R-ACTCTGCTAATTTCTTC GTCCAG; $\beta$-catenin: F-AAAGCGGCTGTTAGTCACTGG, R-CGAGTCATTGC ATACTGTCCAT; e-cadherin: F-CGAGAGCTACACGTTCACGG, R-GGGTGTCGAGGG AAAAATAGG; lactase: F-TCCACCGCTGGTCCTCTAA, R-CACTGTTTTCTCGT CTGGATTCT.

We first analyzed the dissolution curve to determine the specificity of the qPCR amplification. Quantification of the relative expression levels of these target genes was obtained by the formula $2^{-\Delta \Delta \mathrm{Ct}}$, where $\Delta \Delta \mathrm{Ct}=(\mathrm{Ct}$ of the target gene - $\mathrm{Ct}$ of U6) $)_{\text {treatment }}$ - (Ct of the target gene - Ct of U6) $)_{\text {control }}$. Each treatment condition was evaluated in five wells. Data were given in arbitrary units relative to the control, which was assigned a value of 1 .

Western blot analysis. Total protein was harvested by lysate cells with RIPA buffer (Cell Signaling Technologies, Beverly, MA, USA). The bicinchoninic acid assay was used to determine the protein concentration. Proteins were separated by SDS-PAGE gel, and then transferred to a PVDF membrane (Millipore, Billerica, MA, USA). After blocking with 5\% non-fat milk, the membranes were incubated with corresponding primary antibodies in a TBS/Tween-20 buffer overnight at $4^{\circ} \mathrm{C}$. The primary antibodies were against QKI (Santa Cruz Biotechnology, Inc., Santa Cruz, CA, USA), $\beta$-catenin, e-cadherin and lactase (all from Cell Signaling Technologies). Glyceraldehyde 3-phosphate dehydrogenase (GAPDH) was used as an endogenous control. The next day, after washing three times with TBS/Tween-20, the PVDF membranes were incubated with appropriate secondary antibodies for $1 \mathrm{~h}$ at room temperature. Finally, they were washed another three times, then the membranes were soaked in an enhanced chemiluminescence (ECL) reagent for $5 \mathrm{~min}$. Immunoreactivity was detected with the ECL reaction (Pierce Biotechnology, Rockford, IL, USA). All experiments were repeated three times.

Luciferase reporter assay. The wild-type (WT) 3'UTR and mutant (Mut) 3'UTR of the human QKI gene were cloned according to the instructions from http://www.targetscan.org/. The primers used to amplify the QKI gene were as follows: QKI-3'UTR F-5'-CACGTCTAGAGCATGTGTTT GACCTG-3' and QKI-3'UTR R-5'-GGACTCCGGAGC GCATACCAGTAA GCACG-3'. Then the reporter vector was constructed to the PGL3-basic vector (Promega Corporation, Madison, WI, USA), and the cloning site was $X b a \mathrm{I} / X b a \mathrm{I}$. The SW480 cells were cultured in 12-well plates. When the cells reached $80-90 \%$ confluence, the cells were transfected with $0.75 \mu \mathrm{g}$ PGL3-WT-3'UTR-QKI and PGL3-Mut-3'UTR-QKI plasmids, and then 10 pmol mimic-miR-155 or mimic-control (Ruibobio, Guangzhou, China) was added using Lipofectamine 2000 (Invitrogen Life Technologies, Carlsbad, CA, USA). After $24 \mathrm{~h}$, the cells were harvested by digestion. The luciferase activity was measured according to the manufacturer's instructions. The relative luciferase activity was expressed as the ratio of firefly luciferase fluorescence to renilla luciferase fluorescence.

Cell cycle assay. The SW480 cells were cultured in DMEM in 12 -well plates. When they reached $80 \%$ confluence, miR-155 and QKI vector was added to the supernatant. Following stimulation for $24 \mathrm{~h}$, the SW480 cells were harvested through centrifugation, and then fixed with a 70\% ethanol ice bath for $24 \mathrm{~h}$. The following day, the cells were washed with cold phosphate-buffered saline twice, and then stained with propidium iodide (PI) protected from the light at $37^{\circ} \mathrm{C}$ for $30 \mathrm{~min}$. Each treatment condition was investigated in three wells. Then flow cytometry (BD Biosciences, San Jose, CA, USA) was used to measure the cell number in the G1, S and G2 phases.

Cell scratch assay. For scratch assays, the SW480 cells (cell density $5 \times 10^{5} / \mathrm{cm}^{2}$ ) were spread in a capsule. Then the cells were stimulated with various treatments. After overspreading the entire field of the microscope without overlapping, certain cells were wiped off to leave a cross-shaped area without cells. After $24 \mathrm{~h}$, the results were evaluated according to the coverage of cells.

Statistical analysis. GraphPad 5.0 software (GraphPad Software Inc., San Diego, CA, USA) was used to process the data, which are presented as the means \pm standard deviation. Comparisons between two groups were performed with a two-tailed t-test. $\mathrm{P}<0.05$ was considered to indicate a statistically significant difference.

\section{Results}

High expression of miR-155 and low expression of QKI in colon cancer tissues. The qPCR findings revealed higher miR-155 expression and lower QKI expression in colon cancer tissue than in normal gastric intestinal epithelial tissue (Fig. 1). 




Figure 1. Expression of microRNA-155 (miR-155) and quaking (QKI) in colon cancer tissues. The expression of miR-155 increased significantly in colon cancer tissues compared with normal tissues. Furthermore, colon cancer tissues with lymph node metastasis (LM) had a higher expression level of miR-155 than tissues with no lymph node metastasis (NLM). In contrast, QKI mRNA expression was lower in colon cancer tissues than in normal tissues, and lower in tissues with lymph node metastasis compared with tissues without lymph node metastasis. ${ }^{*} \mathrm{P}<0.05,{ }^{* *} \mathrm{P}<0.01$, compared with miR-155 expression; ${ }^{*} \mathrm{P}<0.05$, compared with QKI expression.

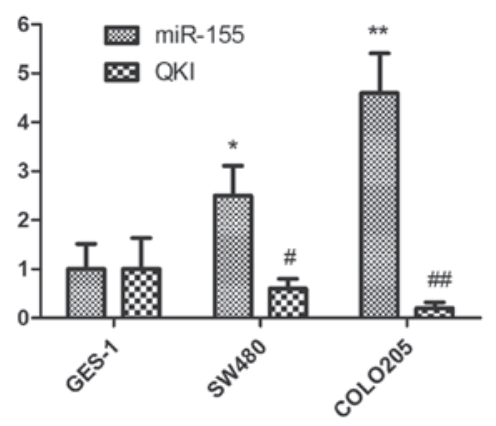

Figure 2. Expression of microRNA-155 (miR-155) in colon cancer cell lines SW480 and COLO205. In accordance with the clinical results, the results of quantitative polymerase chain reaction demonstrated that miR-155 expression was higher in SW480 and COLO205 than in GES-1. Moreover, COLO205 expressed a higher level of miR-155 compared with SW480. ${ }^{*} \mathrm{P}<0.05,{ }^{* *} \mathrm{P}<0.01$, compared with miR-155 expression; ${ }^{~} \mathrm{P}<0.05,{ }^{\# \#} \mathrm{P}<0.01$, compared with QKI expression.

Moreover, colon cancer tissues with lymph node metastasis had higher miR-155 expression than the cancer tissues without lymph node metastasis (Fig. 1). Correspondingly, in comparison with the primary colon cancer tissues, lower QKI expression was observed in the colon cancer tissues with lymph node metastasis (Fig. 1).

High expression of miR-155 and low expression of QKI in colon cancer cell lines. As expected, between the SW480 and COLO205 colon cancer cell lines, COLO205 had the stronger invasion and metastasis potential. GES-1 was considered to be the normal control of the two cell lines. qPCR was also performed to investigate the expression level of miR-155 and QKI in colon cancer cell lines. Consistent with the results in cancer tissues, we demonstrated that miR-155 and QKI had a similar expression profile to the colon cancer tissues (Fig. 2), with COLO205 cells having a higher expression level of miR-155 and lower expression level of QKI (Fig. 2).

miR-155 decreases production of QKI by acting on 3'UTR of QKI gene. Due to the phenomenon of the simultaneous existence of a high expression level of miR-155 and a low expression level of QKI in colon cancer, we speculated that QKI was the target of miR-155. By using computational deductions (10), we identified that one miR-155 binding site was included in the $3^{\prime}$ UTR of QKI mRNA. We then designed luciferase reporters with the WT 3'UTR and Mut 3'UTR of QKI (Fig. 3A). The relative luciferase activity of the 3'UTR was decreased by $\sim 45 \%$ when SW480 cells were transfected with mimic-miR-155 (Fig. 3B). Furthermore, when the cells were treated with mimic-miR-155, the QKI expression was significantly decreased at both the mRNA and protein level (Fig. 3C and D). When they were treated with miR-155 inhibitor, the expression of QKI increased significantly (Fig. 3C and D).

miR-155 affects the cell cycle of colon cancer cells in a QKI-dependent manner. In order to verify whether miR-155 affects the colon cancer cell cycle by targeting QKI we firstly performed PI staining of SW480 cells, and the flow cytometry findings revealed that the ratio of $\mathrm{G} 1 / \mathrm{S}$ was increased in SW480 cells when treated with miR-155 (Fig. 4B). When treated with the QKI expression vector, the G1/S ratio also increased significantly (Fig. 4D).

Then, we measured the expression of $\beta$-catenin, which plays a critical role in the cell cycle of cancer cells by promoting the expression of the majority of cell cycle-related genes $(11,12)$. We observed that miR-155 inhibitor decreased the expression of $\beta$-catenin, which was consistent with the results of overexpression of QKI stimulation (Fig. 4E).

miR-155 suppresses the invasion capabilities of SW480 cells and inhibits the expression of e-cadherin and lactase by targeting QKI. A cell scratch assay was carried out to confirm that miR-155 indeed promotes the invasion capabilities of colon cancer cells (Fig. 5B). E-cadherin, a cell adhesion-related protein (13) whose expression is suppressed in colon cancer cells, and lactase, a differentiation marker of colon cells which is expressed at a low level in colon cancer cells, both play significant roles in the differentiation of colon cancer cells $(9,14)$. Treatment with the miR-155 inhibitor and QKI overexpression vector increased the expression of e-cadherin and lactase, which promoted colon cancer cell differentiation; the results obtained through transfection of miR-155 inhibitor were similar (Fig. 5E).

\section{Discussion}

In a previous study, high expression of miR-155 was detected in colon cancer and this was considered to be associated with lymph node metastases (6). To our knowledge, the cellular and molecular effects of miR-155 on colon cancer cells have not previously been reported in detail. In this study, we demonstrated that the RNA-binding protein QKI is a target of miR-155 in colon cancer cells. miR-155 promotes the proliferation and invasion abilities of colon cancer cells by targeting QKI. 
A

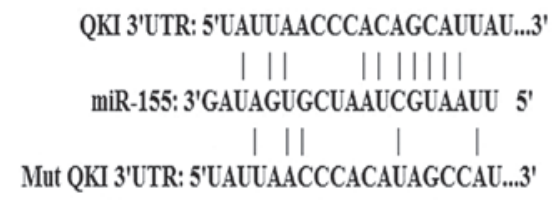

C

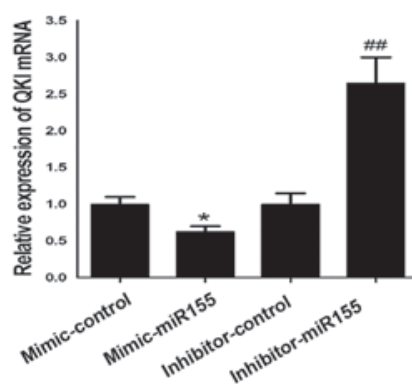

B

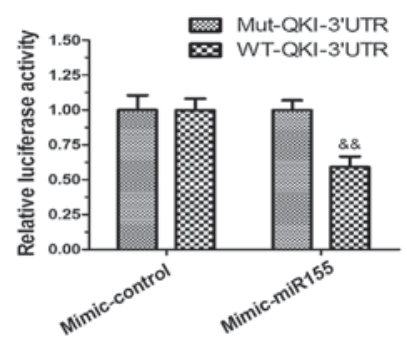

D

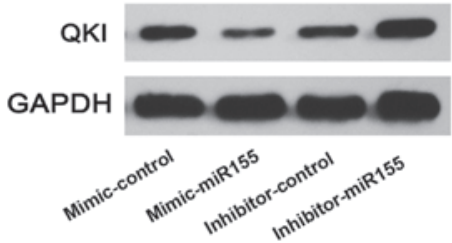

Figure 3. Quaking (QKI) is a target of microRNA-155 (miR-155) in colon cancer. A mutative mRNA binding sequence of miR-155 in the 3 ' untranslated region (UTR) of QKI mRNA and mutated sequence was constructed (A). Relative luciferase activity was decreased when wild-type (WT) 3'UTR-QKI was treated with mimic-miR-155, while the relative luciferase activity of mutant (Mut) 3'UTR-QKI was not affected by the same treatment (B). Expression of QKI was increased significantly following miR-155 inhibitor treatment, and decreased following mimic-miR-155 treatment at the mRNA (C) and protein (D) level. ${ }^{\&}{ }^{2} \mathrm{P}<0.01$, comparing relative luciferase activity between WT-3'UTR-QKI and Mut-3'UTR-QKI treated with mimic-miR-155; ${ }^{*} \mathrm{P}<0.05$, comparing mimic-control group and mimic-miR-155 group; ${ }^{\sharp} \mathrm{P}<0.05$, comparing inhibitor control group and inhibitor miR-155 group.

A

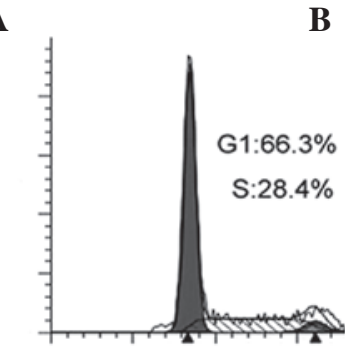

C

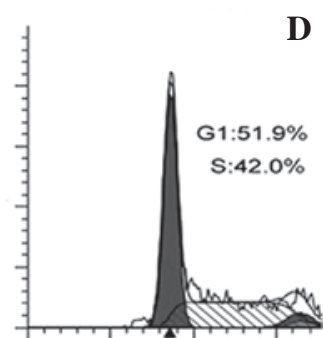

D

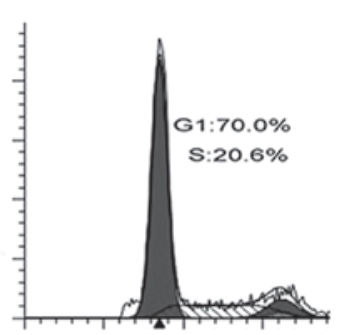

E

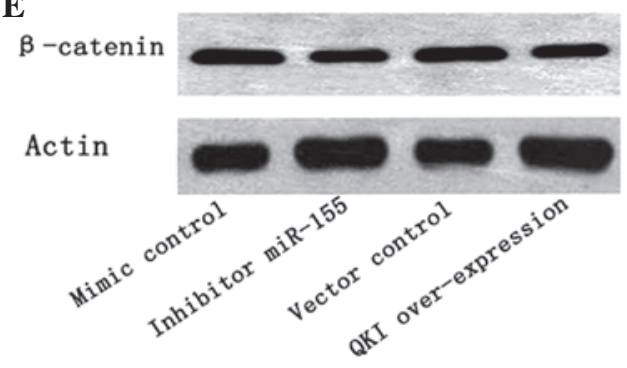

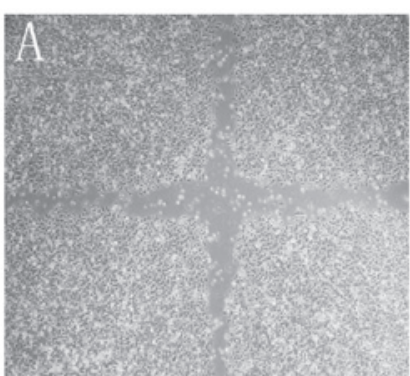
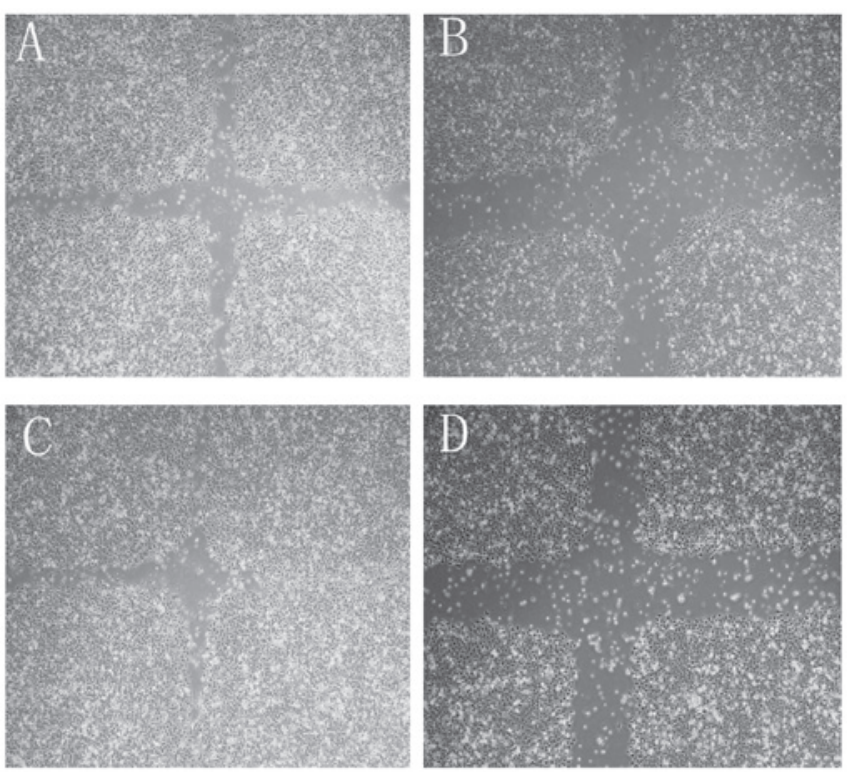

$\mathbf{E}$

e-cadherin-1

Lactase

Actin

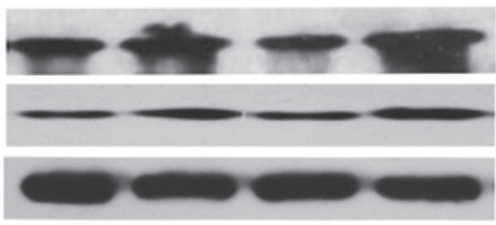

Figure 5. microRNA-155 (miR-155) affects the invasion capability of colon cancer cells by targeting quaking (QKI). The cell scratch assay revealed that after $24 \mathrm{~h}$ the SW480 cells covered the area marked with a cross completely (A), while for cells treated with the miR-155 inhibitor, the thickness of the cross remained relatively large (B). Similarly, compared with the vector control (C), the QKI expression vector inhibited the invasion capacity of SW480 (D). E-cadherin and lactase expression when treated with the miR-155 inhibitor or QKI expression vector (E). 
As is already known, miRNA is mostly relevant to inflammation, and establishes an association between inflammation and cancer (15). Recent studies have shown that high expression of miR-155 was detected in various cancers including breast (16,17), lung (18), lymphoma and leukemia (19). By inducing mutator activity, miR-155 intensified the progression process from normal epithelia cells to cancer cells (20). The present study demonstrated that miR-155 expression is positively correlated with lymph node metastasis in colon cancer, which is consistent with the results of a previous study (6). Furthermore, our study clarified the connection between miR-155 and QKI, which exerts anti-proliferative and differentiative functions in gastric and colon cancer $(8,9)$. In accordance with previous studies $(6,8,9,20)$, our results demonstrated that the low expression of QKI is due to the high expression of miR-155.

In addition, we revealed that inhibiting the function of miR-155 restored the expression of QKI. Furthermore, both overexpression of QKI and inhibition of miR-155 have the same effect on the cell proliferation and invasion abilities of colon cancer cells. In order to confirm the effect of miR-155 on the role of invasion abilities of colon cancer cells, we also analyzed the expression of e-cadherin and lactase, two enterocyte markers of colon epithelial cells. The expression levels of both markers were increased by overexpression of QKI, whose effects are reached uniformly through the inhibition of miR-155. The high expression of $\beta$-catenin detected in colon cancer promotes the proliferation of colon cancer cells and can be suppressed by miR-320a (21). In our results, the expression of $\beta$-catenin was intensively suppressed by miR-155 inhibition and QKI overexpression, while QKI was considered to be a suppressor of $\beta$-catenin.

In conclusion, the present study has revealed that miR-155 regulates the cell cycle and invasion ability of colon cancer cells via the modulation of QKI expression. The study provides novel therapeutic strategies for colon cancer therapy.

\section{Acknowledgements}

The authors would like to thank everyone who assisted in this study.

\section{References}

1. Fearon ER and Vogelstein B: A genetic model for colorectal tumorigenesis. Cell 61: 759-767, 1990.
2. Bushati $\mathrm{N}$ and Cohen SM: microRNA functions. Annu Rev Cell Dev Biol 23: 175-205, 2007.

3. Calin GA and Croce CM: MicroRNA signatures in human cancers. Nat Rev Cancer 6: 857-866, 2006.

4. Croce CM: Causes and consequences of microRNA dysregulation in cancer. Nat Rev Genet 10: 704-714, 2009.

5. Volinia S, Calin GA, Liu CG, et al: A microRNA expression signature of human solid tumors defines cancer gene targets. Proc Natl Acad Sci USA 103: 2257-2261, 2006.

6. Shibuya H, Iinuma H, Shimada R, Horiuchi A and Watanabe T: Clinicopathological and prognostic value of microRNA-21 and microRNA-155 in colorectal cancer. Oncology 79: 313-320, 2011.

7. Iorio MV, Ferracin M, Liu C-G, et al: MicroRNA gene expression deregulation in human breast cancer. Cancer Res 65: 7065-7070, 2005.

8. Bian Y, Wang L, Lu H, et al: Downregulation of tumor suppressor QKI in gastric cancer and its implication in cancer prognosis. Biochem Biophys Res Commun 422: 187-193, 2012.

9. Yang $\mathrm{G}, \mathrm{Fu} \mathrm{H}$, Zhang $\mathrm{J}$, et al: RNA-binding protein quaking, a critical regulator of colon epithelial differentiation and a suppressor of colon cancer. Gastroenterology 138: 231-240, e1-e5, 2010

10. Thomas M, Lieberman $\mathrm{J}$ and Lal A: Desperately seeking microRNA targets. Nat Struct Mol Biol 17: 1169-1174, 2010.

11. Tetsu $\mathrm{O}$ and McCormick F: Beta-catenin regulates expression of cyclin D1 in colon carcinoma cells. Nature 398: 422-426, 1999.

12. van de Wetering M, Sancho E, Verweij C, et al: The $\beta$-catenin/ TCF-4 complex imposes a crypt progenitor phenotype on colorectal cancer cells. Cell 111: 241-250, 2002.

13. Irby RB and Yeatman TJ: Increased Src activity disrupts cadherin/catenin-mediated homotypic adhesion in human colon cancer and transformed rodent cells. Cancer Res 62: 2669-2674, 2002.

14. Zweibaum A, Laburthe M, Grasset E and Louvard D (eds): Use of cultured cell lines in studies of intestinal cell differentiation and function. In: Comprehensive Physiology. Published online, Wiley-Blackwell, pp223-255, 2011.

15. Tili E, Croce CM and Michaille JJ: miR-155: on the crosstalk between inflammation and cancer. Int Rev Immunol 28: 264-284, 2009.

16. Yan LX, Huang XF, Shao Q, et al: MicroRNA miR-21 overexpression in human breast cancer is associated with advanced clinical stage, lymph node metastasis and patient poor prognosis. RNA 14: 2348-2360, 2008.

17. Jiang S, Zhang HW, Lu MH, et al: MicroRNA-155 functions as an OncomiR in breast cancer by targeting the suppressor of cytokine signaling 1 gene. Cancer Res 70: 3119-3127, 2010.

18. Donnem T, Eklo K, Berg T, et al: Prognostic impact of MiR-155 in non-small cell lung cancer evaluated by in situ hybridization. J Transl Med 9: 1-9, 2011.

19. Marcucci G, Maharry KS, Metzeler KH, et al: Clinical role of microRNAs in cytogenetically normal acute myeloid leukemia: miR-155 upregulation independently identifies high-risk patients. J Clin Oncol 31: 2086-2093, 2013.

20. Tili E, Michaille JJ, Wernicke D, et al: Mutator activity induced by microRNA-155 (miR-155) links inflammation and cancer. Proc Natl Acad Sci USA 108: 4908-4913, 2011.

21. Sun JY, Huang Y, Li JP, et al: MicroRNA-320a suppresses human colon cancer cell proliferation by directly targeting $\beta$-catenin. Biochem Biophys Res Commun 420: 787-792, 2012. 